\title{
Effet des techniques de conservation des eaux et des sols, zaï forestier et cordons pierreux, sur la réhabilitation de la végétation herbacée à l'Ouest du Burkina Faso
}

\author{
Jérôme .T. YAMEOGO ${ }^{1 *}$, Mipro HIEN ${ }^{1}$, Anne .M. LYKKE ${ }^{2}$, Antoine .N. SOME ${ }^{1}$ et \\ Adjima THIOMBIANO ${ }^{3}$ \\ ${ }^{1}$ Laboratoire des Systèmes Naturels, des Agro-systèmes et de l'Ingénierie de l'Environnement (Sy.N.A.I.E), \\ Institut du Développement Rural (IDR), Université Polytechnique de Bobo-Dioulasso(UPB). BP : 1091 Bobo- \\ Dioulasso Burkina Faso.E-mail: jerotega@yahoo.fr; ansome30@hotmail.com; miphien@gmail.com \\ ${ }^{2}$ Department of Terrestrial Ecology, NERI National Environmental Research Institute, Aarhus University; \\ Vejlsфvej 25, 8600 Silkeborg, Denmark.E-mail:aml@dmu.dk \\ ${ }^{3}$ Laboratoire de Biologie et Ecologie Végétales, UFR / SVT, Université de Ouagadougou ; 03 BP 7021 \\ Ouagadougou 03, Burkina Faso.E-mail : adjima_thiombiano@yahoo.fr \\ *Auteur correspondant, E-mail : jerotega@yahoo.fr
}

\section{RESUME}

Dans la perspective de trouver des solutions pour remédier au problème de dégradation des ressources naturelles en milieu forestier, des techniques de conservation des eaux et des sols ont été appliquées dans la forêt classée de Kuinima, à l'Ouest du Burkina Faso. L'étude a consisté au suivi de l'évolution de la strate herbacée sur des parcelles où ont été appliquées des techniques de traitement par du zaï forestier et des cordons pierreux. Sur ces parcelles, l'herbe a été récoltée sur des placettes de $1 \mathrm{~m}^{2}$ par la méthode de la récolte intégrale pour suivre l'évolution de la biomasse. Les placettes ont été installées sur les deux diagonales de chaque parcelle. La diversité floristique a été appréciée par un inventaire exhaustif, puis un inventaire stratifié par la méthode des points quadrats. L'étude a révélé un accroissement de la diversité floristique de 6,15 à 35,38\% et une augmentation de la biomasse herbacée de 42,07 à $71,34 \%$ des parcelles aménagées en zaï forestier et en cordons pierreux par rapport aux parcelles témoins. On a noté également un développement des espèces pérennes comme Andropogon gayanus Kunth, Andropogon ascinodis C.B.CI. et Loudetia arundinacea Hochst. ex Steud. dans les espaces aménagés. Ceci témoigne d'une restauration progressive de ces parcelles aménagées.

(C) 2011 International Formulae Group. All rights reserved.

Mots clés: Afrique, diversité végétale, Herbacée, Kuinima, régénération, restauration

\section{INTRODUCTION}

Les changements climatiques et la perte de la diversité biologique sont des problématiques cruciales pour les régions sèches d'Afrique où ces deux phénomènes se combinent pour éprouver les efforts de développement des pays et concomitamment les moyens de vie des populations les plus pauvres de la planète (Hamndou et RequierDesjardins, 2008). Le Burkina Faso compte parmi les pays de ce continent confrontés aux phénomènes de la désertification et du réchauffement climatique (Niang et al., 2004). Les évènements climatiques récents sont un 
exemple frappant de ces impacts sur les populations où les sécheresses des années 1970 et 1980 ont provoqué une famine dramatique et décimé des troupeaux de bovins et de petits ruminants (Sultan et al., 2008). Les changements de la végétation liés aux changements climatiques et à la pression anthropique dans ces pays sont décrits en ce qui concerne la composition spécifique. En outre, la baisse ou la disparition de certaines espèces végétales réduit la couverture du sol et l'expose à l'érosion éolienne et hydrique (Wezel et Haigis, 2000). Ceci concourt à la dégradation du sol et à la détérioration des ressources naturelles. En effet, les sécheresses à répétitions, les catastrophes naturelles et la pression démographique ont eu des conséquences négatives sur l'environnement et entrainé, de ce fait, un retard dans le décollage économique de plusieurs régions (Ganaba et al., 2006 ; Sawadogo et al., 2008). Orgeval (2008) prévoit une variabilité interannuelle des pluies plus accrue pour l'Afrique de l'Ouest pour les années à venir avec de nombreux épisodes de sécheresse locale et une concentration des pluies sur une région plus étroite.

Il est donc nécessaire de protéger les ressources forestières pour conserver la biodiversité et pour satisfaire les besoins des populations dans les zones à vocation essentiellement pastorale où la recherche de la sécurité alimentaire reste une préoccupation. Pour lutter contre la dégradation du sol, diverses techniques d'économie d'eau dont les techniques de conservation des eaux et des sols (CES) sont appliquées en milieu agricole et pastoral (Ganaba, 2005; Kiéma, 2008; Nyssen et al., 2009 ; Zougmoré et al., 2009). La plupart sont des techniques ancestrales améliorées comme c'est le cas des alignements d'obstacles à l'écoulement des eaux de ruissellement (cordons pierreux) et le zaï forestier. Par contre d'autres sont des techniques nouvellement introduites par les structures d'encadrement et de promotion du monde rural (demi-lunes, digues filtrantes, sous-solage). Dans la partie Sud du Burkina Faso, ces techniques sont très peu connues.
Celles qui sont appliquées relèvent d'un simple transfert des technologies expérimentées dans les zones subdésertiques et sahéliennes. Cependant de nos jours on constate une détérioration du couvert végétal de la région Ouest du fait de l'exploitation démesurée des terres et de la baisse de la pluviométrie. Alors survient une inversion des tendances où les forêts font place progressivement à l'agriculture de subsistance (Yaméogo et al., 2009). Les effets de l'action de l'homme, qu'elle soit consciente ou pas, ont influé négativement sur le milieu naturel dans de nombreux cas, entraînant ainsi une fragilité des écosystèmes forestiers et agricoles. Les zones nues, érodées et dégradées se sont multipliées.

L'objectif de cette étude est d'évaluer la contribution des aménagements antiérosifs (zaï forestier et cordons pierreux) sur la régénération herbacée, le premier maillon visible du processus de récupération des sols dégradés.

\section{MATERIELS ET METHODES Milieu d'étude}

La forêt classée de Kuinima est le site de cette étude. Elle est située à l'Ouest du Burkina Faso, entre $04^{\circ} 19$ et $04^{\circ} 36$ de longitude ouest, $11^{\circ} 03$ et $11^{\circ} 07$ de latitude nord. En 1935, elle avait une superficie de 4 000 ha. Actuellement elle ne couvre plus que 2150 ha, une partie ayant été déclassée le 31 mai 1947. La zone d'étude se situe dans le domaine sud-soudanien (Guinko, 1984). La moyenne pluviométrique des dix dernières années (1999-2008) est de $1000 \mathrm{~mm}( \pm 298)$. La zone est caractérisée par l'alternance de deux saisons fortement contrastées: une saison des pluies, de mai à novembre (7 mois) et une saison sèche de décembre à avril (5 mois).

\section{Méthode d'étude \\ Dispositif expérimental}

Le dispositif expérimental forestier mis en place pour l'étude de la végétation est un bloc Fisher complètement randomisé avec deux répétitions (Figure 1). Le dispositif se 
compose de deux parcelles de chacun des traitements suivant: cordons pierreux (CP), zaï forestier $(\mathrm{ZF})$ et témoin $(\mathrm{T})$. Au total, ce dispositif comporte 6 parcelles de $50 \mathrm{~m} \times 50$ $m$ réparties en deux blocs. Les aménagements ont été réalisés en mai 2008. Le dispositif expérimental est sur un sol ferrugineux lessivé induré et la pente est en moyenne de $5 \%$.

\section{Zaïforestier}

Cuvettes de 10 à $15 \mathrm{~cm}$ de profondeur et de 20 à $40 \mathrm{~cm}$ de largeur en saison sèche. La terre sortie de la cuvette est mise en croissant en aval de la cuvette de manière à retenir l'eau de ruissellement.

\section{Cordon pierreux}

Alignement judicieux de moellons suivant les courbes de niveau. Les rangés de cordons pierreux sont espacés d'envirron 33 $\mathrm{m}$.

\section{Témoin}

Parcelle sans aucun aménagement.

\section{Collecte des données}

Dans un premier temps l'inventaire des espèces végétales a été fait de manière exhaustive sur chacune des parcelles. Toutes les espèces herbacées qui ont été rencontrées dans les parcelles ont été inventoriées.

Le suivi de la dynamique de la végétation herbacée a été fait par la méthode des points quadrats (Daget et Poissonet, 1971 ; Mahamane et al., 2007) suivant un réseau de lignes de $20 \mathrm{~m}$ matérialisées par des piquets métalliques (Somé,1996; Kiéma, 2008). La méthode a consisté à tendre un ruban au dessus du toit du tapis herbacé et à effectuer une lecture verticale tous les $20 \mathrm{~cm}$ tout le long à l'aide d'une tige métallique à bout effilé. A chaque point de lecture et le long du bout effilé de la tige, tous les contacts avec les herbes sont pris en compte. L'intérêt de l'étude de la structure de la strate herbacée par point-contact est qu'elle permet une caractérisation très précise et quantifiée de l'état des milieux soumis à l'action de l'homme ou à la pâture par les herbivores domestiques ou sauvages. De même avec cette méthode, il est possible de comparer les formations végétales herbacées entre les différents traitements. Sur des lignes permanentes de relevé, lorsque les mesures de recouvrement par point-contact sont répétées plusieurs années consécutives, il est possible de tirer une conclusion sur l'évolution des formations végétales étudiées (Kiéma, 2007). Nous avons distingué 4 strates : la strate 0-20 $\mathrm{cm}$, la strate $20-50 \mathrm{~cm}$, la strate $50-100 \mathrm{~cm}$ et la strate $>100 \mathrm{~cm}$. Pour les parcelles en cordons pierreux, les lignes ont été placées successivement en amont, au milieu et en aval des cordons.

La biomasse herbacée est estimée sur chaque parcelle dans 5 placettes de $1 \mathrm{~m}^{2}$ chacun par la méthode de la récolte intégrale (Akpo et Grouzis, 2004 ; Rakotoarimanana et al., 2008 ; Walter et Calvo, 2009). Les placettes sont disposées sur les deux diagonales de chaque parcelle. Le poids de l'herbe fauchée sur chaque placette a été pris. Un échantillon représentatif d'environ $500 \mathrm{~g}$ a été prélevé pour la détermination de la matière sèche (MS) à $105{ }^{\circ} \mathrm{C}$ à l'étuve pendant 24 heures.

\section{Analyse des données}

Les données collectées ont permis de calculer :

- La contribution spécifique $(\mathrm{CS} i)$ de chaque espèce qui est définie comme le rapport de la fréquence spécifique (FSi) de cette espèce à la somme des FS de toutes les espèces recensées sur 100 points échantillonnés.

$$
C S i=\frac{F S i}{\sum_{i}^{n} F S i} \times 100
$$

avec $\mathrm{n}=$ le nombre des espèces

Où $\mathrm{CSi}$ et FSi sont les contributions et les fréquences de l'espèce $i$.

- La capacité de charge (CC) est la quantité de bétail que peut supporter un pâturage sans se dégrader, le bétail devant rester en bon état d'entretien, voire prendre du poids ou produire du lait pendant son séjour sur le pâturage (Boudet, 1984). La capacité de charge a été calculée selon la formule de Boudet (1991) sur la base des phytomasses consommables. 
$C C=\frac{P P(\mathrm{~kg} \text { de } \mathrm{MS} / \mathrm{ha}) \times \mathrm{U}}{6,25 \times P U \text { (jour) }}$

$\mathrm{CC}=$ Capacité de charge $; \mathrm{PP}=$ Production de la phytomasse ; $\mathrm{U}=$ Taux d'utilisation (estimé à 40\%, pour la zone sahélienne) ;

6,25 = consommation de l'UBT en $\mathrm{Kg}$ de matière sèche par jour; $\mathrm{PU}=$ Période d'utilisation ;

UBT = Unité Bétail Tropical

- Le coefficient de similitude retenu pour apprécier le degré de ressemblance de la végétation entre les différents traitements est l'index de similitude de Jaccard (ISJ) par ce qu'il tient compte uniquement de la présence des espèces dans la communauté étudiée, ce qui est primordial pour une étude de la diversité. L'ISJ varie de 0 (lorsqu'il n'existe aucune espèce en commun) à 1 lorsque les deux listes sont floristiquement identiques. Mais, pour mieux appréhender la notion, on peut aussi l'exprimer en pourcentage. Cet index est alors donné par le rapport suivant :

$$
I S J=\frac{c}{A+B-c} \times 100
$$

$\mathrm{c}=$ nombre d'espèces communes à la liste 1 et à la liste $2 ; \mathrm{A}=$ nombre d'espèces de la liste $1 ; \mathrm{B}=$ nombre d'espèces de la liste 2 . Les listes 1 et 2 d'espèces représentent successivement celles des deux traitements à comparer (Kiéma, 2008).

Le logiciel JMP a été utilisé pour la séparation des moyennes au seuil de 5\% selon le test de Newman Keuls.

\section{RESULTATS}

\section{Composition floristique}

Les différentes techniques ont eu des effets significatifs sur la dynamique de la plupart des principales espèces. En deuxième année d'aménagement, la richesse floristique herbacée d'ensemble du site ou diversité alpha ( $\alpha$ ) selon la terminologie de Whittaker (1972) se compose de 107 espèces réparties dans 26 familles et 72 genres (Tableau 1). Les familles les plus représentées sont les Poaceae (33\%), les Fabaceae (12\%), les Rubiaceae (8\%), les Cyperaceae (5\%), les Malvaceae $(5 \%)$ et les
Asteraceae $(5 \%)$. Le nombre d'espèces est très variable dans les différentes familles et varie également d'un traitement à un autre. Le traitement $\mathrm{ZF}$ a permis un accroissement de $27,54 \%$ et de $35,38 \%$ respectivement par rapport au $\mathrm{T}$ et aux $\mathrm{CP}$. Les espèces se répartissent ainsi dans les différents aménagements : $\quad 81,31 \%$ des espèces inventoriées sont rencontrées dans le $\mathrm{ZF}$; $63,89 \%$ dans les CP et 60,19\% dans le T. Les Gramineae ou Poaceae regroupent 34 espèces et constituent la famille la plus répandue. Trente et un (31) espèces pérennes sont recensées dans le traitement $\mathrm{ZF}$ contre 25 dans le CP et 24 dans le T. De façon spécifique, 23 espèces sont rencontrées dans le ZF, 7 dans le CP contre 6 dans le T (Tableau 1).

Les index de similitude révèlent une différence de la végétation des différents traitements comparés deux à deux. La similitude est de $56 \%$ entre ZF et CP, 52\% entre $\mathrm{ZF}$ et $\mathrm{T}$ et de $61 \%$ entre $\mathrm{CP}$ et $\mathrm{T}$.

\section{Contribution spécifiques des espèces productives (CS>5\%)}

Les observations montrent que les aménagements ont particulièrement favorisé la présence de Cochlospermum tinctorium Perr., Andropogon ascinodis C.B.CI., Andropogon gayanus Kunth., Andropogon pseudapricus Stapf., Diheteropogon hagerupii Hitchc., Loudetia simplex (Nees) C.E Hubbard, Loudetia togoensis (Pilger) C.E Hubbard, Rhytachne gracilis Stapf., Spermacoce stachydea DC. (Tableau 2). Lorsque l'on compare les traitements au T, on se rend compte que le nombre d'espèces productives augmente dans les traitements. Ainsi, ce nombre passe de 5 espèces dans le $\mathrm{T}$ à 8 espèces dans le $\mathrm{CP}$ et le $\mathrm{ZF}$. En outre, Loudetia togoensis (Pilger) C.E Hubbard régresse dans le $\mathrm{CP}$ et le $\mathrm{ZF}$ au profit des espèces comme Andropogon ascinodis C.B.CI., Andropogon gayanus Kunth., avec toutefois une régression beaucoup plus marquée dans le ZF. ! 
Variation verticale de la diversité
floristique

La végétation herbacée est plus diversifiée dans la première strate $(0-20 \mathrm{~cm})$ pour tous les traitements (Figure 2). En outre le Témoin reste le moins diversifié dans toutes les strates. L'analyse de variance révèle une différence significative du nombre d'espèces entre les deux traitements $(\mathrm{CP} ; \mathrm{ZF})$ et le $\mathrm{T}$ au seuil de 5\% dans toutes les strates. La strate 0 $20 \mathrm{~cm}$ est dominée par des annuelles et des vivaces comme Loudetia togoensis (Pilger) C.E Hubbard, Elionurus elegans Kunth, Sporobolus granularis Mez, Spermacoce stachydea DC., Brachiaria distichophylla Stapf., Cochlospermum planchonii Hook.f. tandis que la strate $>100 \mathrm{~cm}$ est celle des vivaces telles que: Andropogon gayanus Kunth, Andropogon ascinodis C.B.CI. et Loudetia arundinacea Hochst. ex Steud.

\section{Structure verticale du couvert végétal}

L'analyse des pyramides (Figure 3) montre une différenciation de structure en fonction des strates et en fonction des traitements. Ainsi, la somme des nombres de points contacts est beaucoup plus élevée dans le $\mathrm{ZF}$ comparativement aux $\mathrm{CP}$ et au $\mathrm{T}$. Les augmentations sont de l'ordre de $24 \%$ et de $17 \%$ respectivement par rapport aux $\mathrm{CP}$ et au
T. Si l'on considère les strates individuellement, on constate que la strate III $(50-100 \mathrm{~cm})$ concentre plus de biovolumes dans tous les traitements. Elle représente à elle seule $30 \%, 34 \%$ et $36 \%$ des points contacts respectivement au niveau du $\mathrm{ZF}$, des $\mathrm{CP}$ et du T. Le traitement ZF est le seul qui possède une pyramide à base élargie. La dernière strate $(>100 \mathrm{~cm})$ est la moins fournie en points contacts. Néanmoins les traitements ZF et $\mathrm{CP}$ enregistrent des valeurs très élevées par rapport au $\mathrm{T}$.

\section{Production de biomasse au mètre carré}

L'évaluation de la phytomasse montre au Tableau 3 que les aménagements ont permis un développement significatif $(\mathrm{P}<$ $0,05)$ de la végétation. Cela s'est traduit par une augmentation de la production moyenne des parcelles aménagées en ZF de 193,07 g de $\mathrm{MS} / \mathrm{m}^{2}$ et de $113,88 \mathrm{~g}$ de $\mathrm{MS} / \mathrm{m}^{2}$ pour les $\mathrm{CP}$ en comparaison au $\mathrm{T}$. La végétation s'est particulièrement développée aux pieds des CP. De l'observation générale, il ressort une variabilité inter annuelle de la production des parcelles aménagées liée à la pluviosité, à l'hétérogénéité et au faible recouvrement de l'unité. Cependant, le ZF et les CP contribuent fortement à réduire cette hétérogénéité.
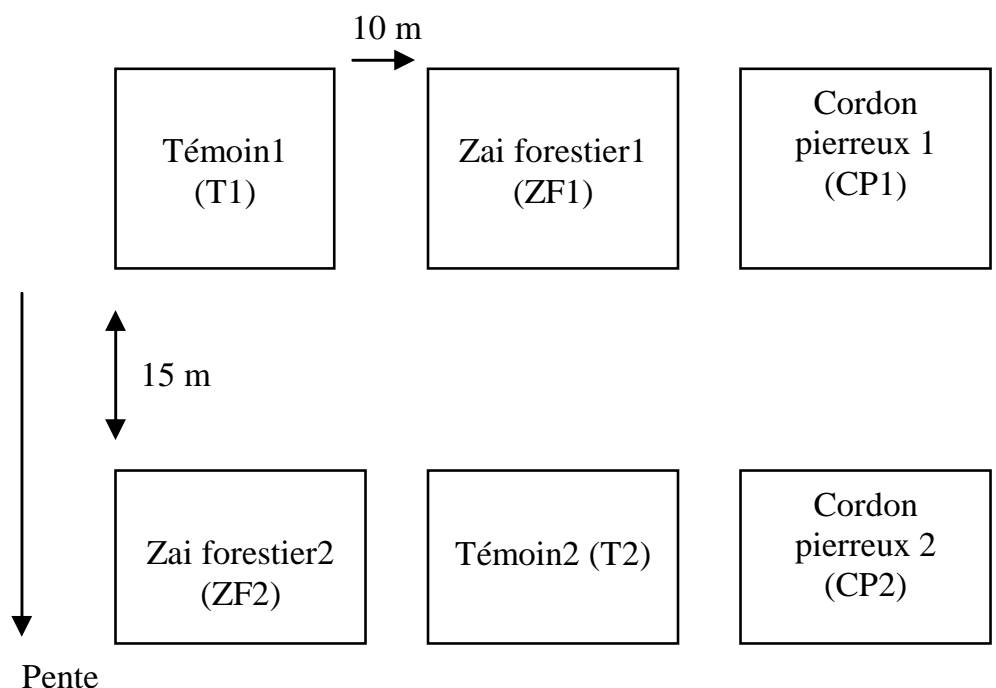

Figure 1: Dispositif expérimental. 


\section{Capacité de charge}

La capacité de charge a été évaluée à partir de la production de phytomasse. Il ressort que les aménagements ont amélioré significativement $(\mathrm{P}<0,05)$ la capacité de charge moyenne de l'unité de végétation de 0,34 UBT pour le ZF et de 0,20 UBT pour le $\mathrm{CP}$ soit des augmentations respectives de $71 \%$ et de $42 \%$ (Tableau 4). Cette augmentation est déjà perceptible à partir de la première année des aménagements où on observe des valeurs respectives de 0,22 UBT et 0,12 UBT pour le $\mathrm{ZF}$ et le $\mathrm{CP}$ et se poursuit même après la deuxième année. Il apparaît cependant de grandes variations inter annuelles dans cette production qui sont probablement liées à la pluviosité et à l'impact de l'activité pastorale. Mais d'un constat général, les aménagements peuvent être considérés comme bénéfiques pour l'amélioration de la production fourragère.

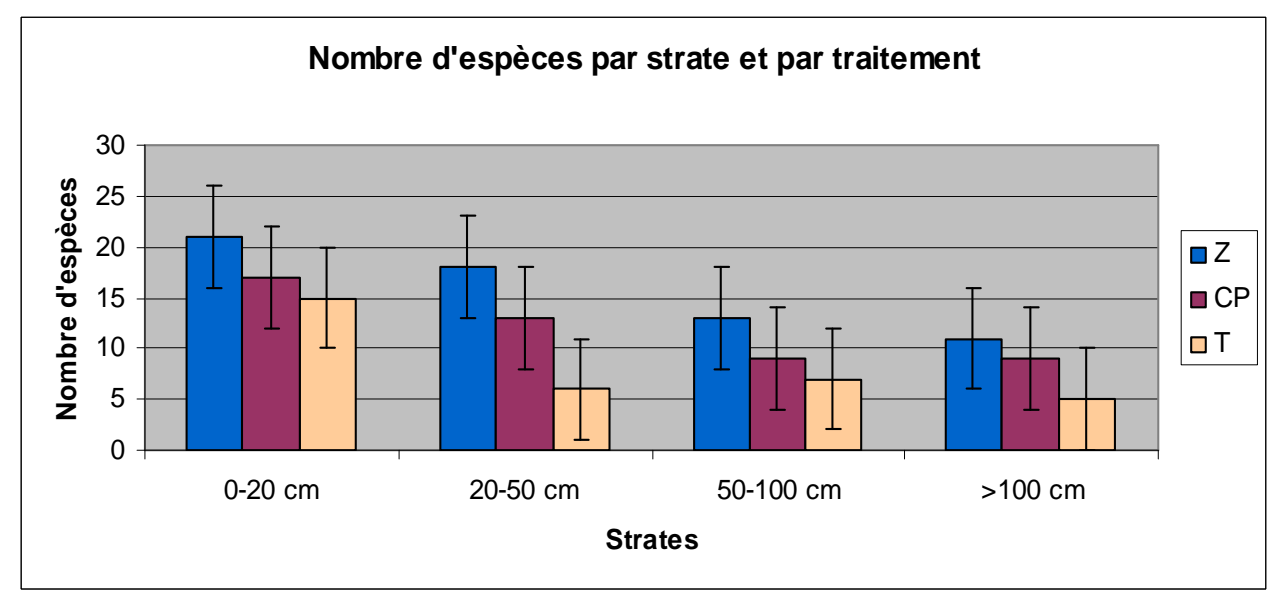

$\mathrm{ZF}=$ Zaï forestier, $\mathrm{CP}=$ Cordon pierreux, $\mathrm{T}=$ Témoin

Figure 2 : Evolution verticale de la diversité floristique.

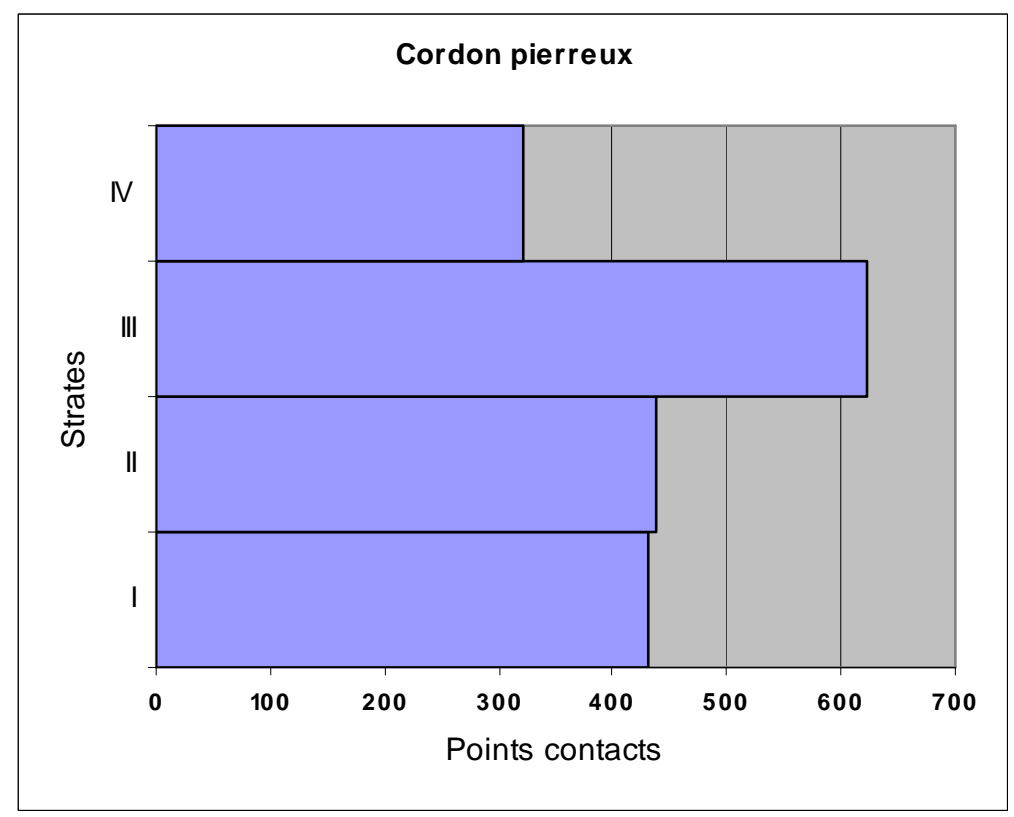



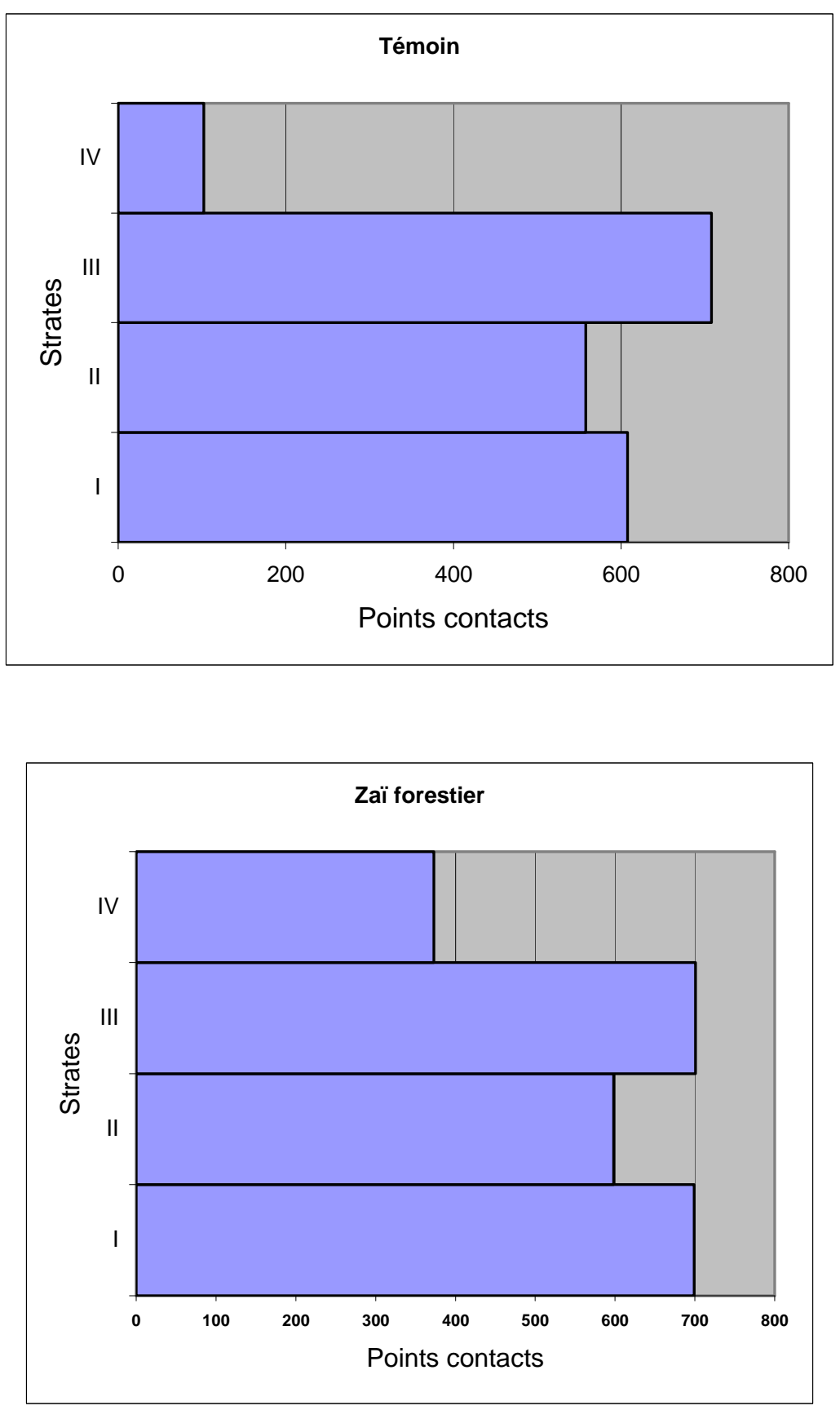

$\mathrm{I}=0-20 \mathrm{~cm} ; \mathrm{II}=20-50 \mathrm{~cm} ; \mathrm{III}=50-100 \mathrm{~cm} ; \mathrm{IV}>100 \mathrm{~cm}$

Figure 3 : Comparaison de la structure verticale des herbacées dans les différents traitements. 
J. T. YAMEOGO et al. / Int. J. Biol. Chem. Sci. 5(1): 56-71, 2011

Tableau 1 : Représentation des herbacées par familles et par espèces dans les traitements en septembre 2009.

\begin{tabular}{|c|c|c|c|c|c|}
\hline \multirow[b]{2}{*}{ Famille } & \multirow[b]{2}{*}{ Genre espèce } & \multirow[b]{2}{*}{ Types biologiques } & \multicolumn{3}{|c|}{ Traitements } \\
\hline & & & $\mathrm{T}$ & $\mathrm{CP}$ & ZF \\
\hline Acanthaceae & $\begin{array}{l}\text { Lepidagathis anobrya Nees } \\
\text { Lepidagathis collina (Endl) Milne-Redh }\end{array}$ & $\begin{array}{l}\mathrm{Hv} \\
\mathrm{Ha}\end{array}$ & + & + & $\begin{array}{l}+ \\
+\end{array}$ \\
\hline \multirow{2}{*}{ Amaranthaceae } & Celosia trigyna Witht ex Steud. & $\mathrm{Ha}$ & + & + & + \\
\hline & Pandiaka heudelotii (Moq) Hiern. & $\mathrm{Ha}$ & + & + & + \\
\hline Araceae & $\begin{array}{l}\text { Stylochaeton warneckei Engl. } \\
\text { Curculigo pilosa (Schum. \& Thonn.) Engl. }\end{array}$ & $\begin{array}{l}\mathrm{Hv} \\
\mathrm{Hv}\end{array}$ & $\begin{array}{l}+ \\
+\end{array}$ & + & + \\
\hline \multirow{4}{*}{ Asteraceae } & $\begin{array}{l}\text { Ageratum conyzoides Sieber ex Steud. } \\
\text { Aspilia kotschyi (Sch.Bip.ex Hochst.) Oliv. }\end{array}$ & $\begin{array}{l}\mathrm{Ha} \\
\mathrm{Ha}\end{array}$ & & & $\begin{array}{l}+ \\
+\end{array}$ \\
\hline & Vernonia nigritiana Oliv. et Hiern. & $\mathrm{Hv}$ & & & + \\
\hline & Vernonia perrottetii Sch. Bip. ex Walp. & $\mathrm{Hv}$ & & + & \\
\hline & Vicoa leptoclada (Webb) Dandy & $\mathrm{Ha}$ & & & + \\
\hline \multirow{2}{*}{ Caesalpiniaceae } & Cassia mimosoides DC. & $\mathrm{Ha}$ & + & + & + \\
\hline & $\begin{array}{l}\text { Cassia occidentalis Naves. } \\
\text { Cassia tora L. }\end{array}$ & $\begin{array}{l}\mathrm{Ha} \\
\mathrm{Ha}\end{array}$ & & + & $\begin{array}{l}+ \\
+\end{array}$ \\
\hline \multirow{2}{*}{ Caryophyllaceae } & Polycarpaea corymbosa (Linn) Lam. & $\mathrm{Ha}$ & + & + & + \\
\hline & Polycarpon prostratum (Forssk.) Asch.\& Schweinf. & $\mathrm{Ha}$ & & & + \\
\hline \multirow{2}{*}{ Cochlospermaceae } & Cochlospermum planchonii Hook.f. & $\mathrm{Hv}$ & + & + & + \\
\hline & Cochlospermum tinctorium Perr. & $\mathrm{Hv}$ & + & + & + \\
\hline \multirow{3}{*}{ Commelinaceae } & Commelina nigritana Benth. & $\mathrm{Ha}$ & & & + \\
\hline & Cyanotis lanata Benth. & $\mathrm{Ha}$ & + & + & + \\
\hline & Cyanotis longifolia Benth. & $\mathrm{Hv}$ & & + & \\
\hline Convolvulaceae & Ipomoea eriocarpa $\mathrm{R} . \mathrm{Br}$ & $\mathrm{Ha}$ & & + & + \\
\hline
\end{tabular}


J. T. YAMEOGO et al. / Int. J. Biol. Chem. Sci. 5(1): 56-71, 2011

\begin{tabular}{|c|c|c|c|c|c|}
\hline Cucurbitaceae & Cucumis melo Blanco. & $\mathrm{Ha}$ & & + & \\
\hline \multirow{6}{*}{ Cyperaceae } & Bulbostylis abortiva C.B.Clarke. & $\mathrm{Ha}$ & + & + & \\
\hline & Cyperus haspan Benth. & $\mathrm{Hv}$ & & & + \\
\hline & Fimbristylis abortiva Steud. & $\mathrm{Ha}$ & & & + \\
\hline & Fimbristylis ferruginea (Linn.) Vahl & $\mathrm{Hv}$ & + & + & + \\
\hline & Fimbristylis hispidula (Vahl.) Kunth & $\mathrm{Ha}$ & + & + & + \\
\hline & Scleria pergracilis Kunth & $\mathrm{Ha}$ & + & + & \\
\hline \multirow{2}{*}{ Euphorbiaceae } & Euphorbia forskalii J. Gay & $\mathrm{Ha}$ & & & + \\
\hline & Sapium grahamii Prain. & $\mathrm{Hv}$ & + & + & + \\
\hline Iridaceae & Gladiolus klatthianus Hutch & $\mathrm{Hv}$ & + & & + \\
\hline \multirow{4}{*}{ Lamiaceae } & Hyptis spicigera Lam. & $\mathrm{Ha}$ & + & + & + \\
\hline & Leucas martinicensis (Jacq.) R.Br & $\mathrm{Ha}$ & + & + & + \\
\hline & Ocimum canum Sims & $\mathrm{Hv}$ & & & + \\
\hline & Tinnea barteri Gürke & $\mathrm{Hv}$ & & + & + \\
\hline \multirow{6}{*}{ Malvaceae } & Hibiscus asper Hook.f. & $\mathrm{Ha}$ & + & + & + \\
\hline & Hibiscus sabdariffa Linn. & $\mathrm{Ha}$ & & + & + \\
\hline & Sida acuta Burm.F & $\mathrm{Hv}$ & + & & + \\
\hline & Sida rhombifolia Linn. & $\mathrm{Hv}$ & & + & + \\
\hline & Sida urens Linn. & $\mathrm{Hv}$ & & + & + \\
\hline & Urena lobata L. & $\mathrm{Hv}$ & & & + \\
\hline Oxalidaceae & Biophytum petersianum Klotzsch. & $\mathrm{Ha}$ & + & + & \\
\hline Fabaceae & Crotalaria senegalensis Bacle & $\mathrm{Ha}$ & & & + \\
\hline
\end{tabular}




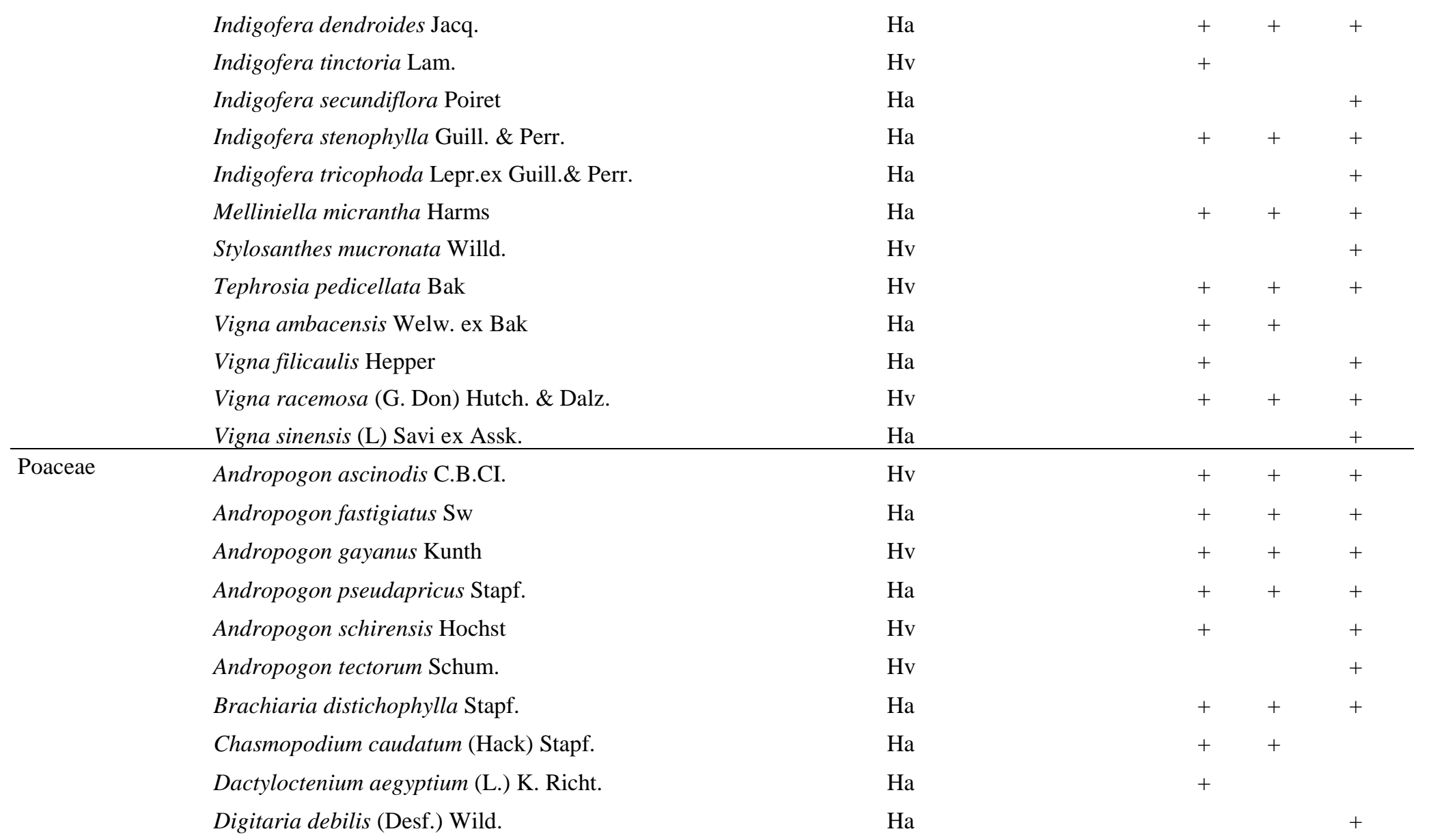


Diheteropogon hagerupii Hitchc.

Eleusine indica Steud.

$\mathrm{Ha}$

Ha

$\mathrm{Hv}$

$\mathrm{Hv}$

Elionurus pobengini Stapf.

Eragrostis cilianensis (All.) Vig.ex Janch.

Eragrostis tremula (L.) Hochst ex Steud.

Hyparrhenia diplandra (Hack.) Stapf.

Loudetia arundinacea Hochst. ex Steud.

Loudetia simplex (Nees) C.E Hubbard

Loudetia togoensis (Pilger) C.E Hubbard

Microchloa indica Druce

Monocymbium ceresiiforme (Nees) Stapf.

Panicum maximum Nees

Paspalum scrobiculatum Linn.

Pennisetum pedicellatum Trin

Rhytachne gracilis Stapf.

Brachiaria distichophylla Stapf.

Schizachyrium exile (Hochst) Pilger.

Schizachyrium platyphyllum

(Franch.) Stapf.

Schizachyrium sanguineum (Retz) Alston

Setaria pallide-fusca (Schumach)

C.E Hubb

$\begin{array}{llll}\text { Ha } & + & + & + \\ \mathrm{Hv} & + & + & + \\ \mathrm{Hv} & + & + & + \\ \mathrm{Ha} & & + & + \\ \mathrm{Ha} & & + & \\ \mathrm{Hv} & & + & + \\ \mathrm{Hv} & + & + & \\ \mathrm{Hv} & + & + & + \\ \mathrm{Ha} & + & + & + \\ \mathrm{Ha} & + & + & + \\ \mathrm{Ha} & + & + & + \\ \mathrm{Ha} & + & + & + \\ \mathrm{Ha} & & & + \\ \mathrm{Ha} & + & + & + \\ \mathrm{Ha} & + & + & + \\ \mathrm{Ha} & & + & \\ \mathrm{Ha} & + & + & + \\ \mathrm{Hv} & & + & \\ \mathrm{Hv} & & + & + \\ \mathrm{Ha} & & & +\end{array}$




\begin{tabular}{|c|c|c|c|c|c|}
\hline & Sporobolus festivus Hochst. ex. A. Riche & $\mathrm{Hv}$ & & + & \\
\hline & Sporobolus pectinellus $\mathrm{Mez}$ & $\mathrm{Ha}$ & & + & + \\
\hline & Tripogon minimus Hochst. ex Steud. & $\mathrm{Ha}$ & + & & \\
\hline Polygalaceae & Polygala arenaria Oliv. & $\mathrm{Ha}$ & + & & + \\
\hline \multirow{9}{*}{ Rubiaceae } & $\begin{array}{l}\text { Fadogia agrestis Schweinf ex Hiern. } \\
\text { Kohautia senegalensis }\end{array}$ & $\mathrm{Hv}$ & + & + & + \\
\hline & Cham. \& Schlecht. & $\mathrm{Ha}$ & + & + & + \\
\hline & Mitracarpus scaber Zucc & $\mathrm{Ha}$ & & + & + \\
\hline & Oldenlandia corymbosa Herb. Madr. ex Witht. \& Arn. & $\mathrm{Ha}$ & + & + & + \\
\hline & Oldenlandia herbacea (Willd.) Roxb. & $\mathrm{Ha}$ & & + & + \\
\hline & Spermacoce filiformis Hiern. & $\mathrm{Ha}$ & + & + & \\
\hline & Spermacoce radiata Sieber ex DC. & $\mathrm{Ha}$ & + & & + \\
\hline & Spermacoce stachydea DC. & $\mathrm{Ha}$ & + & + & + \\
\hline & Spermacoceae filifolia (Schum.\& Thonn) Leburn & $\mathrm{Ha}$ & + & + & + \\
\hline Scrophulariaceae & $\begin{array}{l}\text { Scoparia dulcis L. } \\
\text { Striga hermonthica (Delile) Benth. }\end{array}$ & $\begin{array}{l}\mathrm{Hv} \\
\mathrm{Ha} \\
\end{array}$ & + & + & $\begin{array}{l}+ \\
+ \\
\end{array}$ \\
\hline Sterculiaceae & Waltheria indica Linn. & $\mathrm{Hv}$ & + & + & + \\
\hline \multirow{2}{*}{ Taccaceae } & Tacca leontopetaloides (Linn) Kuntze & $\mathrm{Hv}$ & + & & \\
\hline & Triumfetta rhomboidea Jacq. & $\mathrm{Ha}$ & + & & \\
\hline Tiliaceae & Corchorus tridens Linn. & $\mathrm{Ha}$ & & + & + \\
\hline Zingiberaceae & Kaempferia aethiopica Solms ex Engl. & $\mathrm{Hv}$ & + & + & + \\
\hline \multicolumn{3}{|c|}{ Nombre d'espèces par traitement } & 65 & 69 & 87 \\
\hline \multicolumn{3}{|c|}{ Pourcentage par rapport à l'effectif total } & 60,19 & 63,89 & 81,31 \\
\hline
\end{tabular}


Tableau 2 : Contribution spécifique des espèces productives (\%).

\begin{tabular}{lccc}
\hline Traitements & Zaï forestier & Cordon pierreux & Témoin \\
\hline Cochlospermum tinctorium & - & 6,40 & - \\
Andropogon ascinodis & 25,03 & 15,30 & 6,00 \\
Andropogon gayanus & 6,25 & 6,71 & - \\
Andropogon pseudapricus & 8,16 & 5,63 & 5,45 \\
Diheteropogon hagerupii & 5,02 & 5,67 & 5,12 \\
Loudetia simplex & 15,07 & 5,41 & 5,06 \\
Loudetia togoensis & 22,12 & 39,42 & 66,87 \\
Rhytachne gracilis & 5,00 & - & - \\
Spermacoce stachydea & 7,01 & 5,57 & - \\
Autres & 6,34 & 9,89 & 11,50 \\
\hline
\end{tabular}

Tableau 3 : Dynamique de la production de biomasse (g de $\mathrm{MS} / \mathrm{m}^{2}$ ).

\begin{tabular}{lccc}
\hline Traitements & \multicolumn{2}{c}{ Année } & Moyenne \\
\cline { 2 - 3 } & 2008 & 2009 & \\
\hline Témoin & $282 \mathrm{~b}$ & $259,25 \mathrm{c}$ & $270,63 \mathrm{c}$ \\
Zaï forestier & $410,25 \mathrm{a}$ & $517,14 \mathrm{a}$ & $463,7 \mathrm{a}$ \\
Cordon pierreux & $352,87 \mathrm{a}$ & $416,15 \mathrm{~b}$ & $384,51 \mathrm{~b}$ \\
\hline \multicolumn{2}{l}{ Les moyennes dans la même colonne suivies de la même lettre alphabétique ne diffèrent pas } \\
significativement au seuil de 5\% selon le test de Newman Keuls
\end{tabular}

Tableau 4 : Capacité de charge des différents traitements (UBT/ha/an).

\begin{tabular}{|c|c|c|c|}
\hline \multirow[t]{2}{*}{ Traitements } & \multicolumn{2}{|c|}{ Année } & \multirow[t]{2}{*}{ Moyenne } \\
\hline & 2008 & 2009 & \\
\hline Témoin & $0,4944 c$ & $0,4545 c$ & $0,4744 \mathrm{c}$ \\
\hline Zaï forestier & $0,7193 a$ & $0,9067 \mathrm{a}$ & $0,813 \mathrm{a}$ \\
\hline Cordon pierreux & $0,6187 b$ & $0,7296 b$ & $0,6741 b$ \\
\hline
\end{tabular}

\section{DISCUSSION}

Le nombre de familles, de genres et d'espèces augmente significativement avec les aménagements. Le ZF a permis un accroissement du nombre d'espèces de $35,38 \%$ et le CP de 6,15\% par rapport au T. Ces résultats corroborent ceux trouvés en milieux sahéliens par certains auteurs (Kiéma et al., 2001 ; Kiéma et al., 2007 ; Sawadogo et al., 2008) qui ont tous noté une amélioration de la diversité floristique avec les mesures anti-érosives.

Les index de similitude révèlent aussi une différence de la végétation des différents traitements comparés deux à deux. Cette différence s'explique d'une part par le fait que les $\mathrm{CP}$ et les $\mathrm{ZF}$ constituent des pièges pour les semences et d'autre part du fait qu'ils créent des conditions favorables à la germination de ces semences. La différence a été plus marquée entre le $\mathrm{ZF}$ et $\mathrm{T}(\mathrm{ISj}=52 \%)$. En outre, ce traitement a permis une diversification plus élevée à tous les niveaux de stratification et $79,49 \%$ des herbacées vivaces se rencontre en son sein. A cet effet, Walter et Calvo (2009) qui ont travaillé en Espagne, notaient que l'apport de matière organique permettait un accroissement de la diversité herbacée et de la biomasse. Somé (1996), ayant travaillé sur les jachères, 
concluait que la présence de certaines herbacées vivaces comme les Andropogon spp. était indicatrice de période de remise en culture. Kiéma (2007) ajoute que la proportion des graminées pérennes et annuelles est un bon indicateur de la dynamique et de la capacité de reconstitution des milieux postculturaux. Le ZF permettrait donc une récupération rapide des sols dégradés.

Concernant la contribution spécifique, quelques espèces végétales apparaissent régulièrement et en proportion plus importante. Les $\mathrm{CP}$ et les $\mathrm{ZF}$ favorisent surtout l'installation des espèces hygrophiles en conformité avec l'augmentation de l'humidité du sol et des apports en sédiments. Ces espèces s'installent au détriment d'autres plus tolérantes à la sècheresse. Kiéma (2008) a fait le même constat dans la zone sahélienne du Burkina Faso avec les CP.

La végétation herbacée constitue un élément important dans le processus de restauration des écosystèmes. Elle est de par sa biomasse un stock de matière organique et d'éléments minéraux pour le sol. Ainsi, pour Garnier et Dajoz (2001), la végétation herbacée participe de l'ordre de 75 à $90 \%$ à la biomasse totale dans les savanes tropicales. En colonisant le milieu, cette végétation pionnière crée les conditions favorables à l'installation des autres espèces herbacées et ligneuses. De Blic et Somé (1997) ont montré que les andropogonées vivaces dès leur apparition en touffes isolées jouaient un rôle positif vis-à-vis de la structure et de la porosité de l'horizon superficiel. La résistance à la pénétration racinaire est plus faible et la porosité plus élevée du fait de la structure devenue fragmentaire de cet horizon. Le ZF a enregistré le meilleur rendement en production de biomasse $\left(463,7 \mathrm{~g} \mathrm{MS} / \mathrm{m}^{2}\right)$ et en nombre de points de contacts dans toutes les strates. Ce résultat est le reflet de la densité des poquets de zaï mais traduit également l'existence de conditions favorables au développement de la végétation. La strate III $(50-100 \mathrm{~cm})$ contient plus de biomasse comparativement aux autres strates. En effet, à cette période de la saison «septembre » les inflorescences bien développées des herbacées s'épanouissent dans cette strate.

Après deux années d'aménagement, les effets sur le niveau de production enregistré laissent penser que les CP sont des aménagements durables. Ainsi, il ressort que dès la première année les potentialités liées à l'effet des aménagements s'expriment de façon significative. En revanche les variations inter annuelles sont surtout liées d'une part à la pluviométrie et à l'exploitation pastorale durant la saison des pluies, le site n'étant pas protégé (Sawadogo et al., 2005; Kiéma, 2008) et d'autre part à une amélioration progressive de la fertilité du sol.

La végétation s'est particulièrement développée au pied des $\mathrm{CP}$ et dans les poquets de $\mathrm{ZF}$. Cette hétérogénéité de la répartition de la végétation dans ces aménagements nous permet de dire avec Hien (1997) que l'amont des $\mathrm{CP}$ et les poquets de $\mathrm{ZF}$ accumulent les eaux de ruissellement provenant de l'espace qui sépare chaque ouvrage de celui situé immédiatement vers l'amont. Ces endroits offrent en premier lieu les conditions de germination des semences en début d'hivernage et peuvent à ce titre être considérés comme le «nid de germination». L'aval des CP qui précède immédiatement peut en revanche bénéficier en profondeur, des écoulements hypodermiques latéraux liés à l'accumulation de l'eau en amont. Il est donc plus favorable aux végétaux pérennes à enracinement profond.

Pour ce qui est du ZF, il est le dispositif qui a le plus permis une diversification de la végétation herbacée et une intense activité végétative pendant la saison pluvieuse. Cependant, les poquets de ZF sous l'effet de l'érosion hydrique sont pratiquement bouchés, ce qui probablement les empêcherait à la longue de jouer leur rôle d'accumulateur de matière fertilisante, de semence et d'eau. Le même constat a été fait en 2006 par Ganaba (Ganaba, 2006) dans la zone subsahélienne du Burkina Faso.

Ce travail a permis de montrer que les traitements $\mathrm{ZF}$ et $\mathrm{CP}$ offraient une amélioration significative de la diversité 
floristique, de la biomasse et un meilleur développement des espèces pérennes en zone sud soudanienne du Burkina Faso. Il atteste ainsi qu'avec l'effet du changement climatique et de l'accélération de la dégradation des sols, cette zone qui n'était pas reconnue comme une zone d'applicabilité du zaï (Zombré, 2003), est devenue de plus en plus réceptive à l'utilisation de la technique du ZF, notamment sur les sols ferrugineux tropicaux lessivés. Ce travail pourrait ainsi être un atout pour la restauration des espaces forestiers dans la zone et une clef pour une exploitation durable des zones pastorales.

$$
\text { L'analyse des propriétés }
$$

biophysicochimiques et hydrodynamiques qui feront l'objet de la suite de cette étude à court terme permettra de mieux apprécier les changements opérés dans le sol suite à la mise en place de l'aménagement. A long terme il sera question de l'impact des traitements sur la végétation ligneuse.

\section{REMERCIEMENTS}

Nos remerciements s'adressent au projet SUN (FP6 INCO-dev 031685) qui a entièrement financé ces travaux.

\section{REFERENCES}

Akpo LE, Grouzis M. 2004. Interaction arbre/herbe en bioclimat semi-aride : influence de la pâture. Sécheresse, 15(3): 253-261.

Boudet G. 1984. Manuel sur les Pâturages Tropicaux et Cultures Fourragères. Ministère des relations extérieures, de la coopération et du développement : Institut d'élevage et de médecine vétérinaire des pays tropicaux (IEMVT) ; Collection Manuels et Précis d'Elevage: Montpellier.

Boudet G. 1991. Manuel sur les Pâturages Tropicaux et les Cultures Fourragères (2ème édn). Ministère de la coopération et du développement : Institut d'élevage et de médecine vétérinaire des pays tropicaux (IEMVT); Collection Manuels et Précis d'Elevage: Montpellier.

Daget P, Poissonet J. 1971. Une méthode d'analyse phytologique des prairies, critères d'application. Ann. Agron., 22: 541.

De Blic Ph, Somé NA. 1997. Etat structural d'horizons superficiels sableux sous culture ou jachère herbacée en Afrique de l'Ouest (Burkina Faso). Etude Gest. Sols, 4(1): 17-24.

Ganaba S. 2005. Impact des aménagements de conservation des eaux et des sols sur la régénération des ressources ligneuses en zone sahélienne et nord soudanienne du Burkina Faso. Vertig0, 6(2): 126-140.

Ganaba S, Bayala J, Yaméogo KM, Yaméogo JT, Ouadba JM. 2006. Impact des aménagements anti-érosifs sur la phénologie, la transpiration et la productivité fruitière ligneuses en zone subsahélienne du Burkina Faso. Etudes Flor. Vég. Burkina Faso, 10: 49-62.

Garnier LKM, Dajoz I. 2001. The influence of fire on the demography of a dominant grass species of West African savannas, Hyparrhenia diplandra. J. Ecol., 89(2): 200-208.

Guinko S. 1984. Végétation de la Haute Volta. Thèse de Doctorat, Université Bordeaux III, Bordeaux, p.394.

Hamndou AD, Requier-Desjardins $\mathrm{M}$. 2008. Variabilité climatique, désertification et biodiversité en Afrique : s'adapter, une approche intégrée. VertigO, 1(8). URL: http://vertigo. revues.org/5356.

Hien GF. 1997. Dynamique et productivité des ligneux dans la régénération des terres dégradées en milieu Soudanosahélien. Rev. Sci. Tech., 22(2): 36-44.

Kiéma A. 2008. Effets des techniques de restauration et d'exploitation des pâturages naturelles sahéliens sur la dynamique de la production fourragère. Thèse de doctorat, Université Polytechnique de Bobo-Dioulasso, BoboDioulasso, p.175.

Kiéma A, Nianogo AJ, Kaboré-Zoungrana C. 2007. Étude bilan des aménagements anti-érosifs dans les pâturages en région sahélienne du Burkina Faso. Études Rech. Sahé., 13: 21-28. 
Kiéma A, Ouédraogo T, Nianogo AJ, Sanou S. 2001. Effets des cordons pierreux et du scarifiage sur la régénération des pâturages naturels en région sahélienne du Burkina Faso. Rev. Sci. Tech. Série Sci. Nat. Agron., 25(2): 99-113.

Kiéma S. 2007. Elevage extensif et conservation de la diversité biologique dans les aires protégées de l'Ouest burkinabé. Arrêt sur leur histoire, épreuves de la gestion actuelle, état et dynamique de la végétation. Thèse de doctorat, Université d'Orléans, Orléans, p.562.

Mahamane A, Ichaou A, Ambouta JMK, Mahamane S, Morou B, Amani I, Mahamadou H, d'Herbès JM, Gineste P, Wata I, Issaka A. 2007. Indicateur écologique de mise en culture de jachère au Niger. Sécheresse, 18(4): 289-95.

Niang D, Mermoud A, Yacouba H, Karambiri H. 2004. Fonctionnement hydrique de différents types de formations éoliennes en milieu sahélien burkinabé. Sud Sci. Tech., 12: 4-12.

Nyssen J, Haile M, Naudts J, Munro N, Poesen J, Moeyersons J, Frankl A, Deckers J, Pankhurst R. 2009. Desertification? Northern Ethiopia rephotographed after 140 years. Sci. Total Environ., 407: 2749-2755.

Orgeval T. 2008. Impact du changement climatique sur la saison des pluies en Afrique de l'Ouest : que nous disent les modèles de climat actuels ? Sécheresse, 19(2): 79-85.

Rakotoarimanana V, Gondard H, Ranaivoarivelo N, Carrière S. 2008. Influence du pâturage sur la diversité floristique, la production et la qualité fourragère d'une savane des Hautes Terres malgaches (région de Fianarantsoa). Sécheresse, 19(1): 39-49.

Sawadogo H, Bock L, Lacroix D, Zombré NP. 2008. Restauration des potentialités de sols dégradés à l'aide du Zaï et du compost dans le Yatenga (Burkina Faso).
Biotechnol. Agron. Soc. Environ., 12(3): 279-290.

Sawadogo L, Tiveau D, Nygard R. 2005. Influence of selective tree cutting, livestock and prescribed fire on herbaceous biomass in the savannah woodlands of Burkina Faso, West Africa. Agric. Ecosyst. Environ., 105: 335-345.

Somé NA. 1996. Les systèmes écologiques post-culturaux de la zone soudanienne (Burkina Faso) : structure spatiotemporelle des communautés végétales et évolution des caractères pédologiques. Thèse de Doctorat, Université Pierre et Marie Curie, Paris, p.212.

Sultan B, Janicot S, Baron C, Dingkuhn M, Muller B, Traoré S, Sarr B. 2008. Les impacts agronomiques du climat en Afrique de l'Ouest : une illustration des problèmes majeurs. Sécheresse, 19(1): 29-37.

Walter I, Calvo R. 2009. Biomass production and development of Native Vegetation Following biowaste amendement of a degraded, semi-arid soil. Arid Land Res. Manag., 23: 297-310.

Wezel A, Haigis J. 2000. Farmers perception of vegetation changes in semi-arid Niger. Land Degrad. Develop., 11: 423-34.

Whittaker RH. 1972. Evolution of species diversity. Taxon, 21: 213-251.

Yaméogo TJ, Somé NA, Hien M. 2009. Etude préliminaire à une restauration de sols dégradés en zone soudanienne du Burkina Faso. Sécheresse, 20(1): 32-8.

Zombré NP. 2003. Les sols très dégradés (Zipela) au centre nord du Burkina Faso : Dynamique, caractéristiques morphobiopédologiques et impacts des techniques de restaurations. Thèse de doctorat, Université de Ouagadougou, Ouagadougou, p.146.

Zougmoré R, Mando A, Stroosnijder L. 2009. Soil nutrient and sediment loss as affected by erosion barriers and nutrient source in semi-arid Burkina Faso. Arid Land Res. Manag., 23: 85-101. 